\title{
BMJ Open Effect of tourniquet use on the risk of revision in total knee replacement surgery: an analysis of the National Joint Registry Data Set
}

\author{
Muhamed M Farhan-Alanie (D) , ${ }^{1,2}$ Yujin Lee, ${ }^{1}$ Martin Underwood, ${ }^{1}$ \\ Andrew Metcalfe (D) , ${ }^{1,2}$ Mark J Wilkinson (D) , ${ }^{3}$ Andrew James Price, ${ }^{4}$ \\ Jane Warwick, ${ }^{1}$ Peter David Henry Wall (D) ${ }^{1,2}$
}

To cite: Farhan-Alanie MM, Lee $\mathrm{Y}$, Underwood $\mathrm{M}$, et al. Effect of tourniquet use on the risk of revision in total knee replacement surgery: an analysis of the National Joint Registry Data Set. BMJ Open 2021;11:e045353. doi:10.1136/ bmjopen-2020-045353

- Prepublication history for this paper is available online. To view these files, please visit the journal online (http://dx.doi. org/10.1136/bmjopen-2020045353).

Received 28 September 2020 Revised 16 March 2021 Accepted 19 April 2021

Check for updates

(c) Author(s) (or their employer(s)) 2021. Re-use permitted under CC BY. Published by BMJ.

${ }^{1}$ Clinical Trials Unit, Warwick Medical School, University of Warwick, Coventry, UK ${ }^{2}$ Trauma and Orthopaedics, University Hospitals Coventry and Warwickshire NHS Trust, Coventry, UK

${ }^{3}$ Department of Oncology and Metabolism, The University of Sheffield, Sheffield, UK ${ }^{4}$ Nuffield Department of Orthopaedics, Rheumatology and Musculoskeletal Sciences, University of Oxford, Oxford, UK

Correspondence to Muhamed M Farhan-Alanie; muhamed.farhan-alanie@nhs. net

\section{ABSTRACT}

Objective Tourniquet use in total knee replacement (TKR) is believed to improve the bone-cement interface by reducing bleeding, potentially prolonging implant survival. This study aimed to compare the risk of revision for primary cemented TKR performed with or without a tourniquet.

Design We analysed data from the National Joint Registry (NJR) for all primary cemented TKRs performed in England and Wales between April 2003 and December 2003. Kaplan-Meier plots and Cox regression were used to assess the influence of tourniquet use, age at time of surgery, sex and American Society of Anaesthesiologists (ASA) classification on risk of revision for all-causes.

Results Data were available for 16974 cases of primary cemented TKR, of which 16132 had surgery with a tourniquet and 842 had surgery without a tourniquet. At 10 years, 3.8\% had undergone revision $(95 \% \mathrm{Cl} 2.6 \%$ to $5.5 \%$ ) in the no-tourniquet group and $3.1 \%$ in the tourniquet group ( $95 \% \mathrm{Cl} 2.8 \%$ to $3.4 \%$ ). After adjusting for age at primary surgery, gender and primary ASA score, the HR for all-cause revision for cemented TKR without a tourniquet was 0.82 (95\% $\mathrm{Cl} 0.57$ to 1.18$)$.

Conclusions We did not find evidence that using a tourniquet for primary cemented TKR offers a clinically important or statistically significant reduction in the risk of all-cause revision up to 13 years after surgery. Surgeons should consider this evidence when deciding whether to use a tourniquet for cemented TKR.

\section{BACKGROUND}

Total knee replacement (TKR) is widely used to relieve pain and improve function for individuals with end-stage symptomatic arthritis of the knee. ${ }^{12}$ In the UK, the majority of TKR components are cemented in place to hold and stabilise them in the correct position in the bone. ${ }^{3}$ The surgery can be performed with or without the use of a thigh tourniquet. Survey research has identified that most surgeons in the UK and around the world perform TKR with the aid of a tourniquet and that this practice has remained

\section{Strengths and limitations of this study}

- This is the largest and longest cohort comparing revision for all-causes following total knee replacement (TKR) surgery performed with versus without a tourniquet.

- Approximately 17000 TKR procedures from 384 centres were included in this study, enabling generalisability of our results to similar patient groups.

- We have considered all-cause revision as our primary outcome to mitigate the risk of misclassification of indication for revision.

- Patients were followed up for a median of 12.2 years from their primary procedure, and we have accounted for patient death in our analysis.

- We could not control for confounding factors including tourniquet inflation timing, pressure and duration as these details were not collected in the National Joint Registry minimum dataset and patients were not randomised.

unchanged over time. ${ }^{4-6}$ In 2003, 93\% of primary TKRs were performed with a tourniquet. A 2015 survey of the British Association of Knee Surgeons (BASK) indicated that $90 \%$ of surgeons routinely use a tourniquet. ${ }^{78}$ The reasons for the widespread use of a tourniquet include a belief that bleeding bone surfaces might impair the fixation of cemented prostheses and reduce long-term implant survivorship. ${ }^{7}$ There are data from laboratory animal studies that suggest blood interferes with the strength of the bone-cement interface. ${ }^{9}$ This may translate to an increased risk of aseptic loosening of the implant components, the most common cause of TKR failure necessitating revision surgery. ${ }^{3}$ However, there is also evidence suggesting that tourniquets do not improve implant fixation and longevity. Two randomised controlled trials (RCTs) in humans which used radiostereometric analysis (RSA - a surrogate marker of long-term 
implant survival) demonstrated no significant differences in tibial component migration between TKRs performed with versus without tourniquet at up to twoyears of follow-up. ${ }^{1011}$

Tourniquet use is not without its risks and has been associated with various adverse effects such as increased pain and venous thromboembolism. ${ }^{12}$ They have also been associated with other complications including haematoma formation, wound discharge, skin injuries and nerve palsy, as well as reduced knee range of motion, even two years after surgery. ${ }^{410}$ The routine use of tourniquets in TKR surgery therefore requires scrutiny to determine whether they help to reduce the risk of revision surgery or merely expose patients to unnecessary harm. ${ }^{12}$ This study compares the all-cause revision rate between primary cemented TKR performed with or without tourniquet. If tourniquet use is effective in improving the bone-cement interface then lower rates of revision would be observed in the tourniquet group.

\section{METHODS}

This work forms part of a larger research project titled Safety and Feasibility Evaluation of Tourniquets in Knee Replacement Surgery (SAFE-TKR) and adhered to the published protocol. ${ }^{13}$ This article reports the findings for risk of revision between patients who underwent TKR surgery with versus without a thigh tourniquet. Other outcomes examining potential adverse events and length of hospital will be reported within a separate article.

Data covering England and Wales were provided by the NJR for the period in which tourniquet use was recorded as part of the minimum dataset for knee replacement surgery (April 2003-December 2003). ${ }^{38}$ We analysed only data from primary elective cemented TKRs of which the vast majority were performed for osteoarthritis. ${ }^{8}$ Tourniquet use, along with other routinely collected NJR baseline variables including age at time of surgery, sex and American Society of Anaesthesiologists (ASA) classification, was analysed to measure independent effects, if any, on the rate and timing of all-cause revision.

Kaplan-Meier plots were used to compare time to revision between groups which underwent TKR with and without a tourniquet. Cox regression was performed to quantify the difference between the two groups using hazard ratios (HR) and to assess the effect of the selected baseline variables on risk of all-cause revision. Time to revision was measured from date of index surgery to the earlier of revision and either date of last follow-up or death. All outcomes other than revision were censored. Statistical analyses was performed using STATA V.14 Data and Analysis Software.

\section{Patient and public involvement}

Thirty patients who have undergone TKR were surveyed and took part in a focus group to explore their experiences and views towards tourniquet use during their surgery. Results revealed that patients believed further research on tourniquet use in TKR was important. Implant longevity was identified as being a particular concern among the patients consulted. Patients contributed to the design of this study by helping determine the important outcomes and their timing. The results of this research will be disseminated to study participants through press releases and social media.

\section{RESULTS}

We analysed 20479 primary knee replacement surgery cases done between April 2003 and December 2003. Of the 406 hospitals listed within the NJR system, 384 had returned data $(94 \%) .{ }^{5}$ The numbers undergoing each type of knee replacement surgery, by tourniquet use, are given in table 1 .

Our analysis is of the 16974 cases of primary cemented TKR. Median follow-up was 12.2 years (IQR 8.4-12.6). The baseline characteristics and outcome variables for this subset are shown in tables 2 and 3, respectively.

HRs and associated 95\% CIs from the univariable and multivariable Cox regression models are shown in table 4 . In the univariable model, there was no evidence that the rate at which revisions occur over the study period of 13.3 years differs between those undergoing surgery with a tourniquet and those undergoing surgery without $(\mathrm{HR}=0.83,95 \% \mathrm{CI} 0.57$ to $1.20, \mathrm{p}=0.31)$. Of the selected variables considered, only age at primary surgery and gender were significant in the univariable models. After adjusting for age at primary surgery, gender and

Table 1 Types of knee replacement surgery in the England and Wales NJR dataset (April 2003-December 2003)

\begin{tabular}{lccc}
\hline & $\begin{array}{l}\text { Tourniquet used } \\
(\mathbf{n = 1 9 3 3 0 )}\end{array}$ & $\begin{array}{l}\text { Tourniquet not used } \\
(\mathbf{n = 1 1 4 9 )}\end{array}$ & $\begin{array}{l}\text { Total } \\
\mathbf{( N = 2 0 4 7 9 )}\end{array}$ \\
\hline Type of replacement implant, $\mathrm{n}(\%)$ & & & \\
TKR cemented & $16132(83)$ & $242(73)$ & $16974(83)$ \\
TKR uncemented & $1238(6)$ & $12(1)$ & $1466(7)$ \\
TKR unclassified (eg, hybrid) & $272(1)$ & $57(5)$ & $284(1)$ \\
Unicondylar & $1491(8)$ & $10(1)$ & $207(1)$ \\
\hline Patellofemoral & $197(1)$ & \\
\hline
\end{tabular}

NJR, National Joint Registry; TKR, total knee replacement. 
Table 2 Baseline characteristics for all primary cemented TKRs

\begin{tabular}{|c|c|c|c|}
\hline & $\begin{array}{l}\text { Tourniquet used } \\
(n=16132)\end{array}$ & $\begin{array}{l}\text { Tourniquet not used } \\
(\mathrm{n}=842)\end{array}$ & $\begin{array}{l}\text { Total } \\
(\mathrm{N}=16974)\end{array}$ \\
\hline Age at primary surgery (years), mean (SD) & $71(9)$ & $72(9)$ & $71(9)$ \\
\hline Male & $6871(43)$ & $382(45)$ & $7253(43)$ \\
\hline Female & $9261(57)$ & $460(55)$ & $9721(57)$ \\
\hline \multicolumn{4}{|l|}{ Primary ASA score, $\mathrm{n}(\%)$} \\
\hline P2: mild disease not incapacitating & $9848(61)$ & $508(60)$ & $10356(61)$ \\
\hline P3: incapacitating systemic disease & $1828(11)$ & $97(12)$ & $1925(11)$ \\
\hline P4: life-threatening disease & $35(<1)$ & $2(<1)$ & $37(<1)$ \\
\hline
\end{tabular}

ASA, American Society of Anaesthesiologists; TKR, total knee replacement.

primary ASA grade in the multivariable model, the effect of tourniquet use on the rate at which revisions occur remained largely unchanged $(\mathrm{HR}=0.82,95 \%$ CI 0.57 to $1.18, \mathrm{p}=0.29$ ). Age at primary surgery remained highly significant in the multivariable model and the HRs did not change (global test, $\mathrm{p}<0.001$ ), suggesting that age is both a strong and independent predictor of risk of allcause revision. Gender and primary ASA grade did not reach statistical significance in the multivariable model, suggesting these were not reliable independent predictors for risk of all-cause revision. Figure 1 shows Kaplan-Meier survival curves for time to all-cause revision in patients having TKR surgery with and without a tourniquet.

The difference in percentage unrevised over time (cemented TKR with tourniquet minus cemented TKR without a tourniquet) is presented, together with pointwise confidence intervals, in figure 2 . At 5 years, $2.1 \%$ had undergone revision (95\% CI $1.3 \%$ to $3.3 \%$ ) in the no-tourniquet group and $1.7 \%$ in the tourniquet group (95\% CI $1.49 \%$ to $1.9 \%$ ). At 10 years, $3.8 \%$ had undergone revision (95\% CI $2.6 \%$ to $5.5 \%$ ) in the no-tourniquet group compared with $3.1 \%$ in the tourniquet group (95\% CI $2.8 \%$ to $3.4 \%$ ). Reference lines (indicated red in figure 2) indicate where the magnitude of this difference exceeds $1 \%$. The point estimates of the difference (solid blue line) lie within these two limits throughout. The upper end of the confidence interval does, however, exceed this limit from approximately 3 years onwards. Thus, a difference of more than $1 \%$ in either direction between the tourniquet and no-tourniquet groups cannot be ruled out.

\section{DISCUSSION}

This analysis used data from the world's largest TKR audit dataset, the NJR, and enabled us to examine the association of tourniquet use with all-cause revision surgery following primary, elective cemented TKR. Our results show similar revision rates between people undergoing primary TKR with versus without tourniquet up to 13.3 years after surgery. The only baseline variable studied that showed evidence of an independent effect on the risk of revision was age at primary surgery, consistent with previous research. ${ }^{14}$ We also observed that a tourniquet was used in $95 \%$ of cemented TKRs and $84 \%$ of cementless TKRs. From this, we infer that tourniquet use might

Table 3 Outcome variables for all primary cemented TKRs

\begin{tabular}{|c|c|c|c|}
\hline & $\begin{array}{l}\text { Tourniquet used } \\
(n=16132)\end{array}$ & $\begin{array}{l}\text { Tourniquet not used } \\
(\mathrm{n}=842)\end{array}$ & $\begin{array}{l}\text { Total } \\
(\mathrm{N}=16974)\end{array}$ \\
\hline \multicolumn{4}{|l|}{ Outcome, n (\%) } \\
\hline Revised & $493(3)$ & $30(4)$ & $523(3)$ \\
\hline Unrevised at time of death & $6149(38)$ & $346(41)$ & $6495(38)$ \\
\hline \multicolumn{4}{|l|}{ Timing of death, n (\%) } \\
\hline Within 30 days & $59(<1)$ & $3(<1)$ & $62(<1)$ \\
\hline $31-90$ days & $42(<1)$ & $2(<1)$ & $44(<1)$ \\
\hline After 90 days & $6048(37)$ & $341(40)$ & $6389(38)$ \\
\hline
\end{tabular}

$I Q R$, interquartile range; TKR, total knee replacement. 
Table 4 HRs and associated 95\% Cls from the univariable and multivariable Cox regression models for all-cause revision

\begin{tabular}{|c|c|c|c|c|}
\hline & \multicolumn{2}{|l|}{ Univariable models } & \multicolumn{2}{|l|}{ Multivariable model } \\
\hline & HR $(95 \% \mathrm{Cl})$ & $P$ value & HR (95\% Cl) & $P$ value \\
\hline \multicolumn{5}{|l|}{ Tourniquet used } \\
\hline Yes & $0.83(0.57$ to 1.20$)$ & 0.31 & $0.82(0.57$ to 1.18$)$ & 0.29 \\
\hline Age at primary surgery (years) & & & $0.17(0.11$ to 0.27$)$ & \\
\hline 65-69 & 0.75 (0.60 to 0.94$)$ & & 0.74 (0.60 to 0.93$)$ & \\
\hline $70-79$ & 0.41 (0.33 to 0.50$)$ & & 0.40 (0.32 to 0.49$)$ & \\
\hline$>80$ & $0.18(0.11$ to 0.27$)$ & $<0.001$ & $0.17(0.11$ to 0.27$)$ & $<0.001$ \\
\hline \multicolumn{5}{|l|}{ Patient gender } \\
\hline Female & 1.00 & & 1.0 & \\
\hline $\mathrm{P} 1$ & 1.00 & & 1.00 & \\
\hline P2 & $0.90(0.74$ to 1.10$)$ & & $1.08(0.89$ to 1.31$)$ & \\
\hline P3 & $1.13(0.84$ to 1.51$)$ & & 1.39 (1.04 to 1.87$)$ & \\
\hline P4 & 1.34 (0.19 to 9.54$)$ & 0.39 & $1.61(0.22$ to 11.48$)$ & 0.19 \\
\hline
\end{tabular}

ASA, American Society of Anaesthesiologists.

be greater in cemented procedures because of a perceived association with better cementation quality. However, this association may have arisen by chance.

Our findings indicate that performing TKR with a tourniquet is not associated with any clinically meaningful difference in the risk of revision compared with surgery without a tourniquet. These findings are in keeping with two RCTs of 50 and 60 patients who underwent primary cemented TKR surgery for osteoarthritis with or without the use of a tourniquet. No significant differences in tibial component migration was observed at up to 2 years of follow-up using radiostereometric analysis. ${ }^{10} 11$ However, patient loss to follow-up in both RCTs resulted in inadequate power to detect differences in migration following

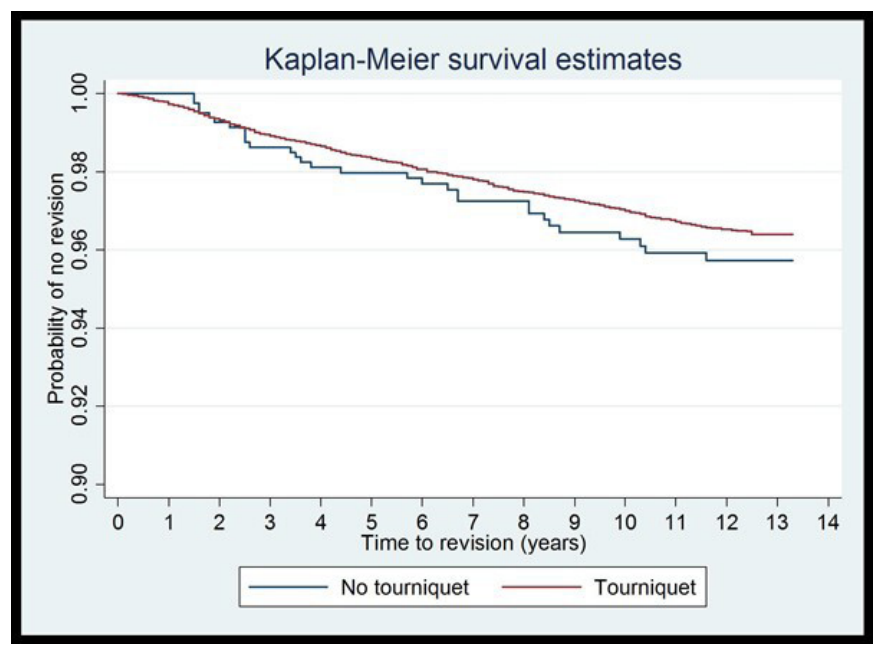

Figure 1 Time to all-cause revision by tourniquet use. oneyear, limiting the validity of the study findings to predict future revision for loosening to between five and ten prosthesis years. ${ }^{15}$ In contrast, our study has compared all-cause revision up to 13.3 years postoperatively and is more reflective of real-life practice as it does not limit surgeons to a defined surgical technique or other factors such as choice of implant or bone cement.

A Cochrane systematic review has shown that using a tourniquet for TKR surgery is associated with an increased risk of serious adverse events which includes venous thromboembolism, infection, reoperation and mortality. Other complications including blisters,

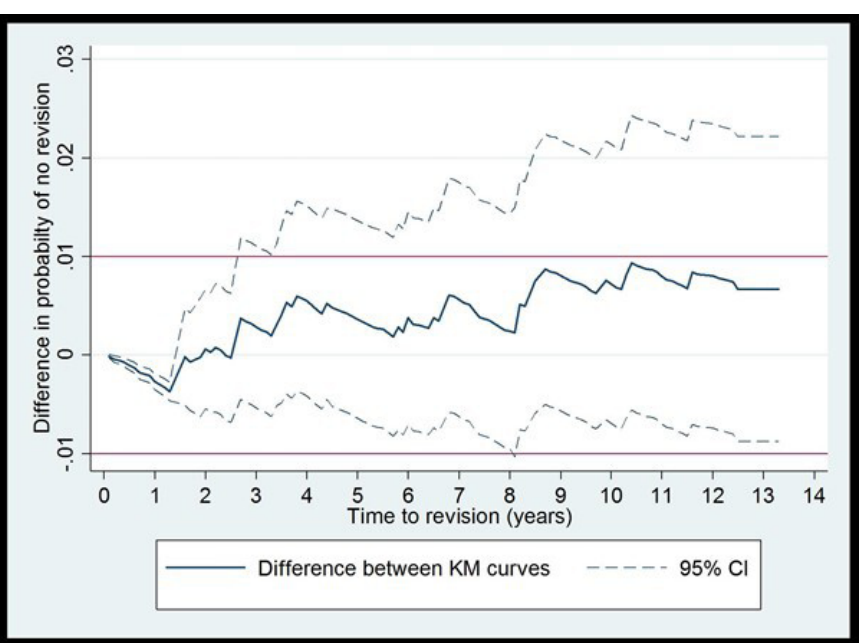

Figure 2 Difference in time to revision (TKR with tourniquet - TKR without tourniquet), over time (unadjusted). TKR, total knee replacement. 
haematoma, wound oozing, bruising and nerve palsy have also been shown to be more common with tourniquet use during TKR surgery, and the devices themselves are frequently colonised with bacteria. ${ }^{4} 1617$ It has been shown that tourniquet use is associated with a modest statistically significant reduction in intraoperative blood loss. This may improve the surgical field of view, but we are not aware of any published literature formally examining this outcome. It is surprising that in the absence of any high-quality evidence demonstrating any likely benefits from using a tourniquet and the existing evidence demonstrating their increased risk of harm to patients, they continue to have such widespread use. Furthermore, there may also be a risk of systemic emboli occurring following the deflation of a tourniquet. ${ }^{18}$ Transcranial Doppler ultrasound studies have found echogenic material in the Circle of Willis after a tourniquet is released and that microembolism can occur even in the absence of a patent foramen ovale. ${ }^{19}$ The potential benefits of tourniquet use during TKR surgery must therefore be balanced against the risks.

We analysed approximately 17000 TKR procedures from over 384 centres which represent $94 \%$ of hospitals listed in the NJR at that time. ${ }^{8}$ Patients were also followed up for a median of 12.2 years from their primary procedure, enabling any potential differences in revision rates to become more apparent particularly as risk of revision for aseptic loosening increases over time. ${ }^{3}$ We have used all-cause revision as the endpoint in our study to mitigate risk of misclassification of aseptic loosening within the NJR. This is a global outcome that considers other failure mechanisms responsible for revision procedure, such as prosthetic joint infection, especially considering that wound complications have been associated with tourniquet usage. ${ }^{40}$ This association is believed to be due to a combination of factors including wound ischaemia and local inflammation. ${ }^{21}{ }^{22}$ The relatively short duration in which tourniquet data was captured within the NJR minimum dataset (April-December 2003) reduces biases by time-dependent unknown variables, such as cementation techniques and polyethylene modification, but does limit the sample size. Conversely, the minimum dataset has evolved over time to include established risk factors for revision such as Body Mass Index which were not captured within version 1 and therefore could not be included in our regression analysis. ${ }^{23}{ }^{24}$ Further limitations also related to application of registry-based data include missing data and under-reporting of procedures, preventing the linkage of revision procedures to any index procedure. ${ }^{325}$ Due to missing data in our dataset, we were not able to perform a subanalysis of revision by indication and assess whether rates of periprosthetic patella fractures and secondary patellar resurfacing differed between the two groups. We acknowledge that we did not consider surgery-related factors such as implant brand, constraint and bearing type in our analysis. This was due to very limited numbers in these subgroups which would not add further meaning to our analysis. Cement brand and viscosity were also not considered, but antibiotic content has not been shown to affect all-cause revision. ${ }^{26}$ However, we have collected data that encompass the diversity of implant designs and cement characteristics across routine practice in the UK, and this helps ensure wide generalisability of the results. Another limitation is the relatively small sample size of TKR procedures performed without a tourniquet across a large number of centres (approximately 150 centres) which may have influenced our results due to performance bias and confounding by indication.

Due to the defined minimum dataset, we could not account for other factors such as use of tranexamic acid and application of hypotensive anaesthesia, which are used to limit bleeding from cancellous bone ends in an attempt to improve the bone-cement interface. Also, variability in tourniquet inflation timing, pressures and duration introduces heterogeneity within the tourniquet group with some surgeons maintaining inflation throughout the entire procedure and others only during the cementation process. However, the study is reflective of real-life routine. Lastly, there is also a risk of bias in our cohort study due to confounding by indication although an RCT to address this is not feasible given the number of participants required to capture small differences in implant survival and the extended follow-up required. Despite the majority of participants (95\%) having surgery with a tourniquet, there were a large number in the no-tourniquet group, and the baseline patient characteristics between the two groups are broadly similar.

\section{CONCLUSIONS}

Our study has shown a similar risk of revision for primary cemented TKRs performed with and without a tourniquet. However, we are unable to exclude conclusively a clinically important improvement in long-term survival for cemented TKRs undertaken with a tourniquet. Surgeons should carefully consider this study's findings as well as the known risks and benefits of tourniquet use, and discuss these issues during the consent process to allow patients to make an informed decision.

\section{Twitter Muhamed M Farhan-Alanie @AlanieMuhamed}

Acknowledgements We thank the patients and staff of all the hospitals who have contributed data to the National Joint Registry (NJR). We are grateful to the Healthcare Quality Improvement Partnership, the NJR Steering Committee and staff at the NJR for facilitating this work. We also thank all patients and public members/ representatives who were involved in this study and, in particular, CG, JS and JD for their ongoing contribution throughout the study.

Contributors MMF-A: drafted and reviewed the final manuscript. MU, AM, MJW and AJP: study design, drafted and reviewed the final manuscript. YL and JW: data analysis, drafted and reviewed the final manuscript. PDHW: study conception, study design, data analysis, drafted and reviewed the final manuscript.

Funding This study presents research funded by a National Institute for Health Research (NIHR) Post-Doctoral Fellowship Award (PDF-2015-08-108).

Competing interests None declared.

Patient consent for publication Not required. 
Ethics approval The project was approved by the National Joint Registry (NJR) Research Committee (May 2016) and the National Research Ethics Committee (January 2016) (15/WM/0455). Patient consent was obtained for data collection by the NJR. According to the specifications of the NHS Health Research Authority, separate informed consent and ethical approval were not required for the present study.

Provenance and peer review Not commissioned; externally peer reviewed.

Data availability statement Access to the data analysed in this study required permission from the National Joint Registry for England, Wales and Northern Ireland Research Sub-committee. http://www.njrcentre.org.uk/njrcentre/Research/ Researchrequests/tabid/305/Default.aspx contains information on research data access request to the National Joint Registry.

Open access This is an open access article distributed in accordance with the Creative Commons Attribution 4.0 Unported (CC BY 4.0) license, which permits others to copy, redistribute, remix, transform and build upon this work for any purpose, provided the original work is properly cited, a link to the licence is given, and indication of whether changes were made. See: https://creativecommons.org/ licenses/by/4.0/.

\section{ORCID iDs}

Muhamed M Farhan-Alanie http://orcid.org/0000-0002-9209-0108

Andrew Metcalfe http://orcid.org/0000-0002-4515-8202

Mark J Wilkinson http://orcid.org/0000-0001-5577-3674

Peter David Henry Wall http://orcid.org/0000-0003-3149-3373

\section{REFERENCES}

1 Wood AM, Brock TM, Heil K, et al. A review on the management of hip and knee osteoarthritis. Int J Chronic Dis 2013;2013:1-10.

2 Bourne RB, Chesworth B, Davis A, et al. Comparing patient outcomes after THA and TKA: is there a difference? Clin Orthop Relat Res 2010;468:542-6.

3 Steering Committee NJR. National joint Registry for England, Wales, Northern Ireland and the Isle of man: 16th annual report. Hemel Hempstead: National Joint Registry Centre, 2019.

4 Zhang W, Li N, Chen S, et al. The effects of a tourniquet used in total knee arthroplasty: a meta-analysis. J Orthop Surg Res 2014;9:13

5 National Joint Registry for England and Wales. National joint Registry for England and Wales 1st annual report 2004.

6 Abdel MP, Berry DJ. Current practice trends in primary hip and knee arthroplasties among members of the American association of hip and knee surgeons: a long-term update. J Arthroplasty 2019;34:S24-7.

7 Gibbs V, Price A, Wall P. Surgical tourniquet use in total knee replacement surgery: a survery of BASK members. Knee 2016;23:III-IV.

8 Steering Committee NJR. National joint Registry for England, Wales, Northern Ireland and the Isle of man: 1st annual report. Hemel Hempstead: National Joint Registry Centre, 2004.

9 Bannister GC, Miles AW. The influence of cementing technique and blood on the strength of the bone-cement interface. Eng Med 1988;17:131-3.
10 Ledin H, Aspenberg P, Good L. Tourniquet use in total knee replacement does not improve fixation, but appears to reduce final range of motion. Acta Orthop 2012;83:499-503.

11 Molt M, Harsten A, Toksvig-Larsen S. The effect of tourniquet use on fixation quality in cemented total knee arthroplasty a prospective randomized clinical controlled RSA trial. Knee 2014;21:396-401.

12 Ahmed I, Chawla A, Underwood M, et al. Tourniquet use for knee replacement surgery. Cochrane Database Syst Rev 2020;12:CD012874.

13 Wall PD, Ahmed I, Metcalfe A, et al. Safety and feasibility evaluation of tourniquets for total knee replacement (SAFE-TKR): study protocol. BMJ Open 2018;8:e022067.

14 Bayliss LE, Culliford D, Monk AP, et al. The effect of patient age at intervention on risk of implant revision after total replacement of the hip or knee: a population-based cohort study. Lancet 2017;389:1424-30.

15 Pijls BG, Valstar ER, Nouta K-A, et al. Early migration of tibial components is associated with late revision: a systematic review and meta-analysis of 21,000 knee arthroplasties. Acta Orthop 2012;83:614-24.

16 Alcelik I, Pollock RD, Sukeik M, et al. A comparison of outcomes with and without a tourniquet in total knee arthroplasty: a systematic review and meta-analysis of randomized controlled trials. $J$ Arthroplasty 2012;27:331-40.

17 Ahmed SMY, Ahmad R, Case R, et al. A study of microbial colonisation of orthopaedic tourniquets. Ann R Coll Surg Engl 2009;91:131-4.

18 Berman AT, Parmet JL, Harding SP, et al. Emboli observed with use of transesophageal echocardiography immediately after tourniquet release during total knee arthroplasty with cement. J Bone Joint Surg Am 1998;80:389-96.

19 Sulek CA, Davies LK, Enneking FK, et al. Cerebral microembolism diagnosed by transcranial Doppler during total knee arthroplasty: correlation with transesophageal echocardiography. Anesthesiology 1999;91:672-6.

20 Garbedian S, Sternheim A, Backstein D. Wound healing problems in total knee arthroplasty. Orthopedics 2011;34:e516-8.

21 Clarke MT, Longstaff L, Edwards D, et al. Tourniquet-Induced wound hypoxia after total knee replacement. J Bone Joint Surg Br 2001;83:40-4.

22 Carden DL, Granger DN. Pathophysiology of ischaemia-reperfusion injury. J Pathol 2000;190:255-66.

23 Namba RS, Cafri G, Khatod M, et al. Risk factors for total knee arthroplasty aseptic revision. J Arthroplasty 2013;28:122-7.

24 Kerkhoffs GMMJ, Servien E, Dunn W, et al. The influence of obesity on the complication rate and outcome of total knee arthroplasty: a meta-analysis and systematic literature review. J Bone Joint Surg Am 2012;94:1839-44.

25 Sabah SA, Henckel J, Cook E, et al. Validation of primary metal-onmetal hip arthroplasties on the National joint Registry for England, Wales and Northern Ireland using data from the London implant retrieval centre: a study using the NJR dataset. Bone Joint $J$ 2015;97-B:10-18.

26 Farhan-Alanie MM, Burnand HG, Whitehouse MR. The effect of antibiotic-loaded bone cement on risk of revision following hip and knee arthroplasty. Bone Joint J 2021;103-B:7-15. 